\title{
Computing Maximal Tori Using LiE and Mathematica
}

\author{
Alfred G. Noël \\ The University of Massachusetts, Department of Mathematics, Boston, MA \\ 02125-3393, USA
}

\begin{abstract}
This paper describes an algorithm for computing maximal tori of the reductive centralizer of a nilpotent element of an exceptional complex symmetric space. It illustrates also a good example of the use of Computer Algebra Systems to help answer important questions in the field of pure mathematics. Such tori play a fundamental rôle in several problems such as: classification of nilpotent orbits of real Lie groups [13], description of admissible nilpotent orbits of real Lie groups [14], [15], [16], [17], classification of spherical nilpotent orbits [8], [9], determination of component groups of centralizers of nilpotents in symmetric spaces [7], [10].
\end{abstract}

\section{Introduction}

A few years ago, while we were at MIT, David Vogan asked us to investigate the classification of admissible orbits of real exceptional Lie Groups. This problem was open for nearly 20 years. After consulting the literature we observed that the problem could be solved by implementing a computational scheme based on a theorem of Takuya Ohta [16]. The interested reader may look at our papers [14],[15] to see a solution. However the same computational scheme can be adapted to several other problems which are part of a long term project which started about seven years ago. As Computer Algebra Systems and computer hardware become more and more powerful we expect them to be a very important part of the pure mathematician's toolbox. In this paper we intend to explain the basic aspects of the algorithmic scheme and to share our thoughts on some software development projects that we shall start in the near future in order to achieve some compatibility between LiE and Mathematica. We hope that pure mathematicians and computer algebra systems designers will find this paper interesting. In order to proceed we need some definitions. The reader who is not familiar with the concepts of Lie algebras and Lie groups or with the notations used below may want to peruse the appendix at the end of this paper.

Let $g$ be a real semisimple Lie algebra with adjoint Lie group $G$ and $g_{\mathbb{C}}$ its complexification. Also let $g=k \oplus p$ be a Cartan decomposition of $g$. Finally, let $\theta$ be the corresponding Cartan involution of $g$ and $\sigma$ be the conjugation of $g_{\mathbb{C}}$ with regard to $g$. Then $g_{\mathbb{C}}=k_{\mathbb{C}} \oplus p_{\mathbb{C}}$ where $k_{\mathbb{C}}$ and $p_{\mathbb{C}}$ are obtained by complexifying 
$k$ and $p$ respectively. Denote by $K_{\mathbb{C}}$ the maximal compact connected subgroup of the adjoint group $G_{\mathbb{C}}$ of $g_{\mathbb{C}}$, with Lie algebra $k_{\mathbb{C}}$.

A triple $(x, e, f)$ in $g_{\mathbb{C}}$ is called a standard triple if $[x, e]=2 e,[x, f]=-2 f$ and $[e, f]=x$. If $x \in k_{\mathbb{C}}, e$ and $f \in p_{\mathbb{C}}$ then $(x, e, f)$ is said to be normal. It is a result of Kostant and Rallis [12] that any nilpotent $e$ of $p_{\mathbb{C}}$ can be embedded in a standard normal triple $(x, e, f)$. Let $k_{\mathbb{C}}(x, e, f)$ be the centralizer of $(x, e, f)$ in $k_{\mathbb{C}}$. Then it is known that $k_{\mathbb{C}}{ }^{(x, e, f)}$ is a reductive Lie algebra. Moreover $k_{\mathbb{C}}{ }^{e}=k_{\mathbb{C}}{ }^{(x, e, f)} \oplus n_{e}$ where $n_{e}$ is a nilpotent ideal.

\section{Problem Description and Algorithm Implementation}

Using the above notations we would like to solve the following problem:

Let $t$ be a Cartan subalgebra of $g_{\mathbb{C}}$ such that $x \in t$. Find a maximal torus $t_{1}$ of $k_{\mathbb{C}}{ }^{(x, e, f)}$ such that $t_{1} \subseteq t$.

The reader should be aware that in general $t_{1} \neq t^{(x, e, f}$. A counterexample can be found in [14]. Furthermore, there is currently no good characterization of such a torus in the literature. And our conversation with several experts led us to believe that such characterization may be quite technical.

Our contribution consists of the solution of the problem for complex exceptional symmetric spaces $p_{\mathbb{C}}$ via an algorithm described below.

From now on we shall assume that the group $G$ is a simple exceptional real Lie group and that $G_{\mathbb{C}} / K_{\mathbb{C}}$ is one of its associated complex symmetric spaces. Let $\Delta$ be the simple Bourbaki root system of $G_{\mathbb{C}}$. If $G$ has equal rank, that is the $\operatorname{rank}(G)=\operatorname{rank}(K)$ then we choose $\Delta_{k}$, a simple root system of $K_{\mathbb{C}}$, to be of Vogan type otherwise we choose $\Delta_{k}$ as in Djoković [5]. The Vogan root systems are described in Knapp [11]. Up to conjugacy we can always chose a representative of a class given in Djoković [4],[5]. Hence it is enough to compute $t_{1}$ from the data given there. To simplify the description of the algorithm we assume that $g$ has equal rank.

\section{Algorithm}

Input: $g$ is a real exceptional simple Lie algebra, $\Delta_{k}=\left\{\beta_{1}, \ldots, \beta_{l}\right\}$, where $l$ is the rank of $k_{\mathbb{C}}$, and $t$ is a Cartan subalgebra of $g_{\mathbb{C}}$ define by $\Delta_{k}$.

\section{Computation}

1. Compute $x$ using the values of $\beta_{i}(x)$. Such values are found in Djoković [4], $[5]$.

2. Using $x$, express $e=\sum_{i=1}^{r} c_{\gamma_{i}} X_{\gamma_{i}}$, where $\gamma_{i}$ is a non compact root, $X_{\gamma_{i}}$ a non zero root vector and $c_{\gamma_{i}}$ a complex number, in one of the regular semisimple 
subalgebra $l_{e}$ of minimal rank $r$ given in Djoković. Create the normal triple $(x, e, f)$

3. Compute the intersection of the kernels of $\gamma_{i}$ on $t$. Observe that the complex span of such an intersection is a maximal torus in $k_{\mathbb{C}}{ }^{(x, e, f)}$.

Output: $t_{1}$ is the complex span of the intersection computed in 3 .

\section{Implementation}

We use two software packages LiE version 1.1 and Mathematica version 4.0 to implement the previous algorithm. The fact that Mathematica offers an environment for symbolic computation makes it possible to solve certain systems of equations in a nice way. We could have used Mapple for that purpose also. We have tried to use some Mathematica packages for Coxeter groups but we were not satisfied with the results. Mathematica [21] is a well known package. LiE is used mostly by mathematicians and physicists who perform computations of a Lie group theoretic nature. LiE does not provide an environment for symbolic computation. However using vectors and matrices with integer entries as basic computational objects it does allow the programmer to access and test a lot of non trivial results and conjectures about complex reductive Lie groups, their representations and their Weyl groups. Moreover, it only works with integer numbers and does not have a polynomial system of equations solver. LiE is written in $\mathrm{C}$ and run mostly on Unix systems. There are also some executables for the classic Macintosh system. More information on Lie can be found in [18]. The computations were carried out on a Power Macintosh G3 running Mac OS 8.6 with 256 Megabytes Random Access Memory. We did not time the algorithm. However familiarity with Lie theory is enough to convince us that most of the modules in LiE should run fast. The only difficulty is the computation of the nilpotent $e$ when the 2-eigenspace of $x$ in $p_{\mathbb{C}}$ is large. We will say more about this below. We shall first give a generic implementation scheme and then we will use a specific example with some pieces of code in order to give the reader a good flavor of the work.

To compute the neutral element $x$, we use the labeling given in Djokokić in order to solve a linear system of equations in Mathematica. Once we have computed $x$, we put its coefficients in a one dimensional array that is fed to a routine written in LiE. This routine computes the 2-eigenspace of $x$ in $p_{\mathbb{C}}$ and returns a list of candidates for $e$. The list of candidates is a two-dimensional array which is reconfigured and fed to Mathematica. This list yields a list of candidates for $f$ by negating its values. At this point Mathematica can test a candidate by solving the system of equations $[e, f]=x$ on the two lists. Lie theory guarantees the existence of a solution. We can now go back to LiE to compute the brackets $\left[H_{\alpha_{i}}, e\right]$ where $\alpha_{i}$ are the simple roots of $g_{\mathbb{C}}$ and $H_{\alpha_{i}} \in t$. This allows us to set up a system of linear equations to be solved in Mathematica. The solution of 
the last system gives a basis for a maximal torus in $k_{\mathbb{C}}{ }^{(x, e, f)}$. This is the desired torus.

\section{Example}

We choose this example so that the reader can quickly check the results. However he or she should be aware that the algorithm can be used for all nilpotents in the exceptional complex symmetric spaces. The reader is encouraged to consult [14],[15]. Let $g$ be $E I I$ (or $E_{6(2)}$ ), a real form of $E_{6}$, and let $\Delta=\left\{\alpha_{1}, \alpha_{2}, \ldots, \alpha_{6}\right\}$ the Bourbaki simple roots of $g_{\mathbb{C}}$. Then $\Delta_{k}=\left\{\beta_{1}, \ldots, \beta_{6}\right\}$, where $\beta_{1}=\alpha_{1}$, $\beta_{2}=\alpha_{3}, \beta_{3}=\alpha_{4}, \beta_{4}=\alpha_{5}, \beta_{5}=\alpha_{6}$ and $\beta_{6}=\alpha_{1}+2 \alpha_{2}+2 \alpha_{3}+3 \alpha_{4}+2 \alpha_{5}+\alpha_{6}$, is a set of simple roots for $k_{\mathbb{C}}=s l_{6}(\mathbb{C}) \oplus s l_{2}(\mathbb{C})$. The root system defined by $\Delta_{k}$ is a Vogan system. See Knapp [11] for more information on such systems.

We consider orbit 6 labeled "00000 4" in Djokovic's classification [4]. We use the given label to compute the neutral element $x$ as follows:

Assume that $x=a H_{\alpha_{1}}+b H_{\alpha_{2}}+c H_{\alpha_{3}}+d H_{\alpha_{4}}+e H_{\alpha_{5}}+f H_{\alpha_{6}}$ with $a, b, c, d, e, f \in$ $\mathbb{Z}$. Using the Cartan matrix of $E_{6}$ we solve the following system in Mathematica:

$\overline{\operatorname{In}[1]:=\text { Solve }[\{2 a-c==0,-a+2 c-d==0,-b-c+2 d-e==0,-d+2 e-f==}$ $0,-e+2 f==0, b==4\},\{a, b, c, d, e, f\}]$

Out[1]: $=\{\{a \rightarrow 2, b \rightarrow 4, c \rightarrow 4, d \rightarrow 6, e \rightarrow 4, f \rightarrow 2\}\}$.

Hence we find $x=2 H_{\alpha_{1}}+4 H_{\alpha_{2}}+4 H_{\alpha_{3}}+6 H_{\alpha_{4}}+4 H_{\alpha_{5}}+2 H_{\alpha_{6}}$.

Next we show how to compute the nilpotent $e$. First, we generate a list of candidates in LiE as follows: ( This is the actual LiE code )

setdefault E6; $\mathrm{n}=$ n_pos_roots; $\mathrm{l}=$ Lie_rank; alpha $=$ pos_roots;

$\# K_{C}$ simple root system \#

beta $=$

[[1,0, $0,0,0,0],[0,0,1,0,0,0],[0,0,0,1,0,0],[0,0,0,0,1,0],[0,0,0,0,0,1],[1,2,2,3,2,1]] ;$

$\mathrm{x}=[2,4,4,6,4,2]$;

\# Store all the roots \#

neg_roots $=-$ alpha; roots $=$ alpha $\wedge$ neg_roots;

for $\mathrm{k}=1$ to $2^{*} n_{-}$pos_roots $\mathrm{do}$

if $(\operatorname{roots}[\mathrm{k}][1] !=1 \& \& \operatorname{roots}[\mathrm{k}][1] !=-1)$ then value $=0$;

for $\mathrm{i}=1$ to $l$ do

for $\mathrm{j}=1$ to $l$ do

value $=$ value $+\operatorname{roots}[\mathrm{k}][\mathrm{i}] * \mathrm{x}[\mathrm{j}] * \operatorname{Cartan}(\operatorname{alpha}[\mathrm{i}]$, alpha[j]); od; od; fi; od;

\# Compute the 2 eigenspace of $\mathrm{x}$ in $p_{\mathbb{C}}$ \#

$\operatorname{pc} 2=0 ;$ peigen $2=\operatorname{null}\left(n \_\right.$pos_roots, 1$)$; 
for $\mathrm{k}=1$ to $2^{*} n \_p o s \_r o o t s$ do

if $(\operatorname{roots}[\mathrm{k}][2]==1\|\| \operatorname{roots}[\mathrm{k}][2]==-1)$ then

pvalue $=0$; for $\mathrm{i}=1$ to $\mathrm{l}$ do for $\mathrm{j}=1$ to $\mathrm{l}$ do

incr $=\operatorname{roots}[\mathrm{k}][\mathrm{i}]{ }^{*} \mathrm{x}[\mathrm{j}] ; \operatorname{prod}=\operatorname{Cartan}(\operatorname{roots}[\mathrm{i}], \operatorname{roots}[\mathrm{j}])$;

pvalue $=$ pvalue + incr* ${ }^{*}$ prod; od; od; print $(\operatorname{roots}[\mathrm{k}]) ; \operatorname{print}($ pvalue $) ;$

if (pvalue $==2$ ) then $\mathrm{pc} 2=\mathrm{pc} 2+1$; peigen2[pc2] = roots[k]; fi; fi; od;

\# Create a list of candidates for $e$ or compute $e$ directly if possible \# count $=0 ; \mathrm{k}=2 ; \mathrm{c}=\operatorname{null}(\mathrm{k})$; for $\mathrm{i}=1$ to $\mathrm{k}$ do $\mathrm{c}[\mathrm{i}]=\mathrm{i}$; od;

$\mathrm{j}=1$;

while $\mathrm{j} !=1$ do if Cartan_type $([$ peigen $2[\mathrm{c}[1]]$,peigen2[c[2]]]) $==\mathrm{A} 2 \mathrm{~T} 4)$

then if $(2([$ peigen $2[\mathrm{c}[1]]+$ peigen $2[\mathrm{c}[2]])=\mathrm{x})$

then for $\mathrm{i}=1$ to $\mathrm{k}$ do print( peigen2[c[i]]); od; print (" ") break;fi;fi;

$\mathrm{j}=\mathrm{k}$;

while $c[j]==(p c 2-k+j)$ do $\mathrm{j}=\mathrm{j}-1$; if $\mathrm{j}==0$ then break fi; od;

if $\mathrm{j} !=0$ then $\mathrm{c}[\mathrm{j}]=\mathrm{c}[\mathrm{j}]+1 \mathrm{fi}$;

for $\mathrm{i}=\mathrm{j}+1$ to $\mathrm{k}$ do if $\mathrm{i}==1$ then $\mathrm{c}[\mathrm{i}]=1$ else $\mathrm{c}[\mathrm{i}]=\mathrm{c}[\mathrm{i}-1]+1$; fi od;od;

The output of the above LiE session is two vectors $[1,1,1,2,1,0]$ and $[0,1,1,1,1,1]$ which are the roots that define

$$
e=\sqrt{2}\left(X_{\alpha_{1}+\alpha_{2}+\alpha_{3}+2 \alpha_{4}+\alpha_{5}}+X_{\alpha_{2}+\alpha_{3}+\alpha_{4}+\alpha_{5}+\alpha_{6}}\right)
$$

and

$$
f=\sqrt{2}\left(X_{-\left(\alpha_{1}+\alpha_{2}+\alpha_{3}+2 \alpha_{4}+\alpha_{5}\right)}+X_{-\left(\alpha_{2}+\alpha_{3}+\alpha_{4}+\alpha_{5}+\alpha_{6}\right)}\right) .
$$

In most cases the equation $[e, f]=x$ has to be solved in Mathematica from the list of candidates in order to determine $e$. Next we evaluate the base elements of $t$ on $e$ in order to set up a system of equations to be solved in Mathematica. The solutions of such a system will give a base of the desired maximal torus. Again we show the implementation in the following $\mathrm{LiE}$ and Mathematica sessions.

$\mathrm{e} 1=[1,1,1,2,1,0] ; \mathrm{e} 2=[0,1,1,1,1,1] ;$

for $\mathrm{i}=1$ to 1 do print (alpha[i]); print (Cartan(e1,alpha[i])); od; print(" ");

for $\mathrm{i}=1$ to 1 do print (alpha[i]); print $(\operatorname{Cartan}(\mathrm{e} 2$,alpha[i] $))$; od;

Since the base elements that we want are of the form $a H_{\alpha_{1}}+b H_{\alpha_{2}}+c H_{\alpha_{3}}+$ $d H_{\alpha_{4}}+e H_{\alpha_{5}}+f H_{\alpha_{6}}$, the output of the above LiE session is fed to Mathematica in the form of the following system of equations.

$\operatorname{In}[2]:=\operatorname{Reduce}[\{a-c+d-f==0,-a+b+c-d+f==0\},\{a, b, c, d, e, f\}$,

Out $[2]:=a==c-d+f \& \& b==0$

This last result gives us a base of the desired maximal torus

$$
\left.t_{1}=\mathbb{C} H_{\alpha_{5}} \oplus \mathbb{C} H_{\alpha_{1}}+H_{\alpha_{3}}\right) \oplus \mathbb{C}\left(H_{\alpha_{4}}+H_{\alpha_{6}}\right) \oplus \mathbb{C}\left(H_{\alpha_{3}}+H_{\alpha_{4}}\right) .
$$




\section{Conclusion and New Directions}

The implementation of the algorithm described above has to be automated further. Currently, LiE and Mathematica do not share data. We had to write translators in order to transfer the different lists to be processed. The implementation of the search for the nilpotent is exhaustive, that is, we try to compute appropriate subsets of a subset of the 2-eigenspace of the neutral element. Such an eigenspace could be large and therefore we need better search techniques in order to quickly eliminate the branches of the search tree which will not take us to a solution. We have started to work in this direction. We can use the theory of Lie groups in order to increase the speed. However we would like to investigate the possibility of using certain search techniques from the field of Computer Science.

Mathematica is a proprietary multi-purpose software package and the source code is not available to the public. This is not the case for LiE which is designed specifically for computations in complex Lie groups and their representations. The mathematical algorithms are well conceived and their implementations seem to work well on average. In some cases where the Weyl group had to be processed, we observed some scalability problems. We are currently investigating the possibility of adding more functions which deal with real Lie groups and their representations. The scope and feasibility of such a project are being evaluated. An other approach is to realize LiE as a Mathematica package. We have not looked at this alternative. However we believed that such package may not work as well as the stand alone version. It is also desirable to design a good graphic user interface for LiE. LiE is maintained by M. A. A. van Leeuwen at l'Université de Poitiers in France. More information on LiE can be obtained at http://wwwmathlabo.univ-poitiers.fr/ maavl/LiE/.

We should point out that John Stembridge from the University of Michigan USA has created a Mapple software package called Coxeter/Weyl for manipulating weights and characters of irreducible representations of semisimple Lie algebras, including functions for computing weight multiplicities, tensor product decompositions, and branching. However, we found $\mathrm{LiE}$ to be faster and easier to use for the type of algorithms we sought to implement. Information on Coxeter/Weyl can be obtained at http://www.math.lsa.umich.edu/ jrs/maple.html\#coxeter.

This work was very enjoyable and continues to help us in our mathematical research either for quickly checking conjectures or for carrying out non trivial computations on Lie groups of moderate sizes. We expect this type of activities to become more prevalent in theoretical investigations. The mathematically inclined reader will find one more interesting application of the algorithm in the following appendix.

Acknowledgment. The author wishes to thank the referees for their helpful and insightful comments. 


\section{References}

1. Auslander L., Kostant B.: Polarization and unitary representations of solvable Lie groups Invent. Math. 14 (1971) 255-354

2. Bourbaki N.: Groupes et Algèbre de Lie Chapitres 4,5,6, Elements de mathématique. MASSON (198)

3. Duflo M.: Construction de représentations unitaires d'un groupe de Lie, Harmonic Analysis and Group Representations, C.I.M.E. (1982)

4. Djoković D.: Classification of nilpotent elements in simple exceptional real Lie algebras of inner type and description of their centralizers, J. Alg. 112 (1987) 577-585

5. Djoković D.: Classification of nilpotent elements in simple real Lie algebras $E_{6(6)}$ and $E_{6(-26)}$ and description of their centralizers J. Alg. 116 (1988) 196-207

6. Kirillov A. A.: Unitary representations of nilpotent Lie groups Russian Math. Surveys $1 \mathbf{1 7}$ (1962) 57-110

7. King D. R.: The Component Groups of Nilpotents in Exceptional Simple Real Lie Algebras Communications In Algebra 120 (1) (1992) 219-284

8. King D. R.: Spherical nilpotent orbits and the Kostant-Sekiguchi correspondence To appear in Trans. Amer. Soc.

9. King D. R.: Classification of spherical nilpotent orbits in complex symmetric space submitted

10. King D. R., Noël A. G.: Component Groups of Centralizers of Nilpotents in Complex Symmetric Spaces Journal of Algebra 232 (2000) 94-125

11. Knapp A. W.:Lie Groups Beyound an Introduction Birkhäuser, Progress in Mathematics Boston 140 (1996)

12. Kostant B., Rallis S.: Orbits and Representations associated with symmetric spaces Amer. J. Math. 93 (1971) 753-809

13. Noel A. G.: Nilpotent orbits and theta-stable parabolic Subalgebras AMS Journal of representation theory $\mathbf{2}$ (1998) 1-32

14. Noël A. G.: Classification of Admissible Nilpotent Orbits In simple Exceptional real Lie algebras of Inner type AMS Journal of Representation Theory 5 (2001) 455-493

15. Noël A. G.: Classification of Admissible Nilpotent Orbits In simple real Lie algebras $E_{6(6)}$ and $E_{6(-26)}$ AMS Journal of Representation Theory 5 (2001) 494-502

16. Ohta T.: Classification of admissible nilpotent orbits in the classical real Lie algebras J. of Algebra 136, No. 1 (1991) 290-333

17. Schwartz J.: The determination of the admissible nilpotent orbits in real classical groups Ph. D. Thesis M.I.T. Cambridge, MA (1987)

18. Van Leeuwen M. A. A., Cohen A. M., Lisser B.:LiE Apackage for Lie Group Computations Computer Algebra Nederland, Amsterdam The Netherlands (1992)

19. Vogan D. jr:Unitary representations of reductive groups Annals of Mathematical Studies, Princeton University Press Study 118 (1987)

20. Vogan D. jr: Associated varieties and unipotent representations Harmonic Analysis on Reductive Groups Birkhäuser, Boston-Basel-Berlin (1991) 315-388

21. Wolfram S.: The Mathematica Book Wolfram media, Cambridge University Press (1998) 


\section{Appendix}

\section{Classification of Real Nilpotent Orbits of $G$ on $g$}

The main result in [13] is a classification of the nilpotent orbits of the real group $G$ on its real Lie algebras $g$. The classification is an extension of the socalled Bala-Carter classification for complex Lie groups. We were able to use the Kostant-Sekiguchi correspondence to classify the nilpotent orbits of $K_{\mathbb{C}}$ on $p_{\mathbb{C}}$ instead. Maintaining the above notations the main result is:

Theorem [Noël]. There is a 1-1 correspondence between triples $(x, e f)$ and triples $(l, q, w)$ where $l$ is a $(\theta, \sigma)$-stable minimal Levi subalgebra of $g_{\mathbb{C}}$ containing $e, q$ a $\theta$-stable parabolic subalgebra of the derived algebra of $l$ and $w$ a $L \cap K_{\mathbb{C}^{-}}$ module in $p_{\mathbb{C}}$. ( $\mathrm{L}$ is a connected Lie group of $g_{\mathbb{C}}$ with Lie algebra $l$ ).

Proof. See [13].

The following proposition gives a construction of the minimal Lie algebra $l$ via the algorithm described in this paper.

Proposition [Noël]. If $l$ is a minimal $(\sigma, \theta)$-stable Levi subalgebra of $g_{\mathbb{T}}$ containing a nilpotent element $e$ of $p_{\mathbb{C}}$ then $l=g_{\mathbb{C}}{ }^{t}$, where $t$ is a maximal toral subalagebra of $k_{\mathbb{C}}^{e}$ and $g_{\mathbb{C}}{ }^{t}$ is the centralizer of $t$ in $g_{\mathbb{C}}$.

Proof. See [13].

The next step in the development of the theory discussed in [13] is the explicit realization of the parametrization given in the preceding theorem. Hence, it is necessary to compute the minimal Levi subalgebra which contains the nilpotent. Here is the LiE session corresponding to the orbit discussed earlier.

\#Compute the minimal Levi contain $e$

\#base of $t$

$\mathrm{t} 1=[0,0,0,0,1,0] ; \mathrm{t} 2=[1,0,1,0,0,0] ; \mathrm{t} 3=[0,0,0,1,0,1] ; \mathrm{t} 4=[0,0,1,1,0,0] ;$

minlev $=\operatorname{null}\left(n_{-}\right.$pos_roots, 1$) ;$ counter $=0$;

for $\mathrm{k}=1$ to $2 n_{-}$pos_roots do if

$\operatorname{Cartan}(\mathrm{t} 1, \operatorname{roots}[\mathrm{k}])==0 \& \& \operatorname{Cartan}(\mathrm{t} 2, \operatorname{roots}[\mathrm{k}])==0 \& \&$

$\operatorname{Cartan}(\mathrm{t} 3, \operatorname{roots}[\mathrm{k}])==0 \& \& \operatorname{Cartan}(\mathrm{t} 4, \operatorname{roots}[\mathrm{k}])==0$ then counter $=$ counter $+1 ; \operatorname{minlev}[\operatorname{counter}]=\operatorname{roots}[\mathrm{k}] ; \mathrm{f} ;$ od;

levy $=$ null (counter,l);

for $\mathrm{k}=1$ to counter do levy $[\mathrm{k}]=\operatorname{minlev}[\mathrm{k}]$;od;

$\operatorname{print}($ Cartan_type(levy));

Thus we find

$$
\begin{aligned}
& {[l, l]=\mathbb{C} X_{ \pm\left(\alpha_{1}+\alpha_{2}+\alpha_{3}+2 \alpha_{4}+\alpha_{5}\right)} \oplus \mathbb{C} X_{ \pm\left(\alpha_{2}+\alpha_{3}+\alpha_{4}+\alpha_{5}+\alpha_{6}\right)} \oplus} \\
& \mathbb{C} X_{ \pm\left(\alpha_{1}+2 \alpha_{2}+2 \alpha_{3}+3 \alpha_{4}+2 \alpha_{5}+\alpha_{6}\right)} .
\end{aligned}
$$


Hence $l$ is of type $A_{2} \oplus T_{4}$. The reader should be aware of the fact that the derived subalgebra of the minimal theta-stable Levi containing the nilpotent is not in general the regular semisimple subalgebra given by Djoković.

\section{Basic Definitions and Notations}

A vector space $g$ over $\mathbb{C}$ or $\mathbb{R}$, with a bilinear bracket operation $[x, y]$ is called a Lie algebra if $[x, x]=0$ and $[x,[y, z]]+[y,[z, x]]+[z,[x, y]]=0$. The set $M_{n}$ of $n \times n$ matrices is a Lie algebra with $[A, B]=A B-B A$ where $\mathrm{A}$ and $\mathrm{B}$ are matrices in $M_{n}$.

A Lie group $G$ is a group and a manifold on which multiplication and taking inverse are analytic. The tangent space of $G$ at the identity element of $G$ is called the Lie algebra of $G$. The set $G L_{n}$ of all $n \times n$ invertible matrices is a Lie group with Lie algebra $M_{n}$.

The classical Lie groups are closed subgroups of $G L_{n}$. The exceptional Lie groups are not subgroups of $G L_{n}$. The same goes for Lie algebras.

A Lie group acts on its Lie algebra. For example, $G L_{n}$ acts on $M_{n}$ by conjugation. The orbits resulting from this action are called conjugacy classes.

A square matrix $B \neq 0$ is nilpotent if there exists $p \in \mathbb{N}$ such that $B^{n}=0$. Each element $z \in g$ defines a map $a d_{z}$ on $g$ as follows: $a d_{z}(y)=[z, y]$ for all $y \in g$. An element $z \in g$ is nilpotent if $a d_{z}$ can be represented by a nilpotent matrix. The conjugacy class of a nilpotent element consists of nilpotent elements and is called a nilpotent orbit.

The set of elements of $G$ which fix a specific element $x \in g$ is called the centralizer of $x$ in $G$ and is denoted by $G^{x}$. The centralizer in $G$ of a subset $S$ of $g$ centralizes every element of $S$ and is denoted by $G^{S}$.

The set of elements $y$ of $g$ which commute with a specific element $x \in g$, that is $[x, y]=0$, is called the centralizer of $x$ in $g$ and is denoted by $g^{x}$. The centralizer in $g$ of a subset $S$ of $g$ commutes with every element of S and is denoted by $g^{S}$.

A real Lie algebra $g$ has a Cartan decomposition $g=k \oplus p$ where $k$ is a compact Lie subalgebra of $g$ and $p$ a vector space. In this case there exists a Cartan involution $\theta$ of $g$ with 1-eigenspace $k$ and (-1)-eigenspace $p$. For example, if $g=\operatorname{sl}(n, \mathbb{R})$, the set of $n \times n$ real matrices of trace zero, then $k=s o(n, \mathbb{R})$ the set of anti-symmetric matrices $g$ and $p$ is made of the symmetric matrices of $g$. In this case the Cartan involution is defined as $\theta(A)=-A^{T}$ for all $A \in g$.

A maximal torus of $g$ is a maximal Abelian subalgebra of $g$. If $g=\operatorname{sl}(n, \mathbb{R})$ then the set of diagonal matrices in $g$ is a maximal torus of $g$. 